15

\title{
Измерения контактной жесткости в атомно-силовом микроскопе
}

\author{
(C) А.В. Анкудинов, ${ }^{1}$ М.М. Халисов ${ }^{2}$ \\ ${ }^{1}$ Физико-технический институт им. А.Ф. Иофрфе РАН, \\ 194021 Санкт-Петербург, Россия \\ ${ }^{2}$ Институт фризиологии им. И.П. Павлова РАН, \\ 199034 Санкт-Петербург, Россия \\ e-mail: alexander.ankudinov@mail.ioffe.ru
}

Поступило в Редакцию 3 апреля 2020 г.

В окончательной редакции 3 апреля 2020 г.

Принято к публикации 3 апреля 2020 г.

\begin{abstract}
Предложен способ повышения точности наномеханических измерений в атомно-силовом микроскопе. Для описания контактного взаимодействия кантилевера с образцом использована аналитическая модель, учитывающая: защемлен зонд кантилевера или скользит по поверхности образца, геометрические и механические характеристики образца и кантилевера, их взаимное расположение. В предположении скольжения разработан фильтр для корректировки сигналов контактной жесткости и деформации, измеряемых на образце с развитым рельефом. Применение фильтра проиллюстрировано на изображениях, полученных в атомносиловом микроскопе с режимом визуализации на базе поточечной регистрации силового квазистатического взаимодействия зонда кантилевера с образцом.
\end{abstract}

Ключевые слова: атомно-силовая микроскопия, кантилевер, скользящий контакт зонд-образец, распределение деформации.

DOI: 10.21883/JTF.2020.11.49989.117-20

\section{Введение}

В атомно-силовой микроскопии (АСМ) сила взаимодействия зонда с образцом определяется по силовым кривым. В новых режимах АСМ - гибридном, HybriD, (NT-MDT SI), PeakForce QNM (Bruker), fast force volume mapping (Asylum Research) - по этим кривым определяются высота рельефа образца и, например, его локальные механические свойства. Существенное влияние на форму силовой кривой может оказывать трение в контакте зонд-образец. Если зонд кантилевера скользит по поверхности, на него действует только нормальная сила. Такая сила изгибает консоль кантилевера, угол изгиба вдоль нее растет монотонно [1]. Когда зонд защемляется на образце значительной силой трения, действующей вдоль поверхности, то консоль может прогнуться (угол прогиба меняется не монотонно) $[1,2]$. В большинстве АСМ-приборов деформации кантилевера регистрируют методом оптического рычага (ОР). Профиль угла отклонения консоли не контролируется, определяется только его значение в одной точке на консоли, в фокусе лазера ОР. Поэтому система управления микроскопа не отличает изгиб от прогиба [3,4], что приводит к ошибочным измерениям амплитуды и направления силы взаимодействия. В схеме ОР измеряются два параметра: углы изгиба, $\alpha$, и кручения, $\beta$, консоли в выбранной точке (рис. 1). Этого не хватает для определения трех проекций вектора смещения точки контакта или приложенной силы. Лишь недавно появилась коммерчески доступная схема регистрации деформаций кантилевера [5], сочетающая
ОР с интерферометром для измерений недостающего третьего параметра - вертикального смещения точки фокуса лазера интерферометра, выбранной на консоли.

В АСМ по относительному наклону $\sigma=S / S_{0}\left(S_{0}-\right.$ средний наклон силовых кривых на условно бесконечно жестком и плоском образце, $S$ - наклон в выбранной на образце точке) и изгибной жесткости консоли $k_{C}$ можно вычислить кажущуюся локальную жесткость $k_{A}$ :

$$
k_{C} / k_{A}=\kappa_{A}^{-1}=\sigma^{-1}-1 .
$$

Связь (1) отвечает простейшей модели взаимодействия кантилевера с образцом в виде двух последовательно соединенных пружин. Не учитывается: защемлен или скользит зонд по образцу, деформация самого зонда, локальный наклон образца и анизотропия его механических свойств, геометрические характеристики и расположение кантилевера над образцом. Строго говоря, вместо локальной нормальной жесткости контакта зонд-образец $k_{S}$ уравнение (1) связывает значения $\sigma \mathrm{c}$ кажущимися параметрами $\kappa_{A}$ или $k_{A}$.

Ранее [6] для повышения точности АCM-измерений $k_{S}$ была предложена более сложная аналитическая модель статического взаимодействия кантилевера в контакте с образцом, которая учитывает перечисленные выше факторы. В АСМ со схемой ОР были измерены углы изгиба и кручения консоли в ответ на смещения в трех ортогональных направлениях тестового образца, контактирующего с зондом. Измерения хорошо согласовывались с моделированием. Для определения жесткости контакта зонд-образец в случае скользящего контакта 

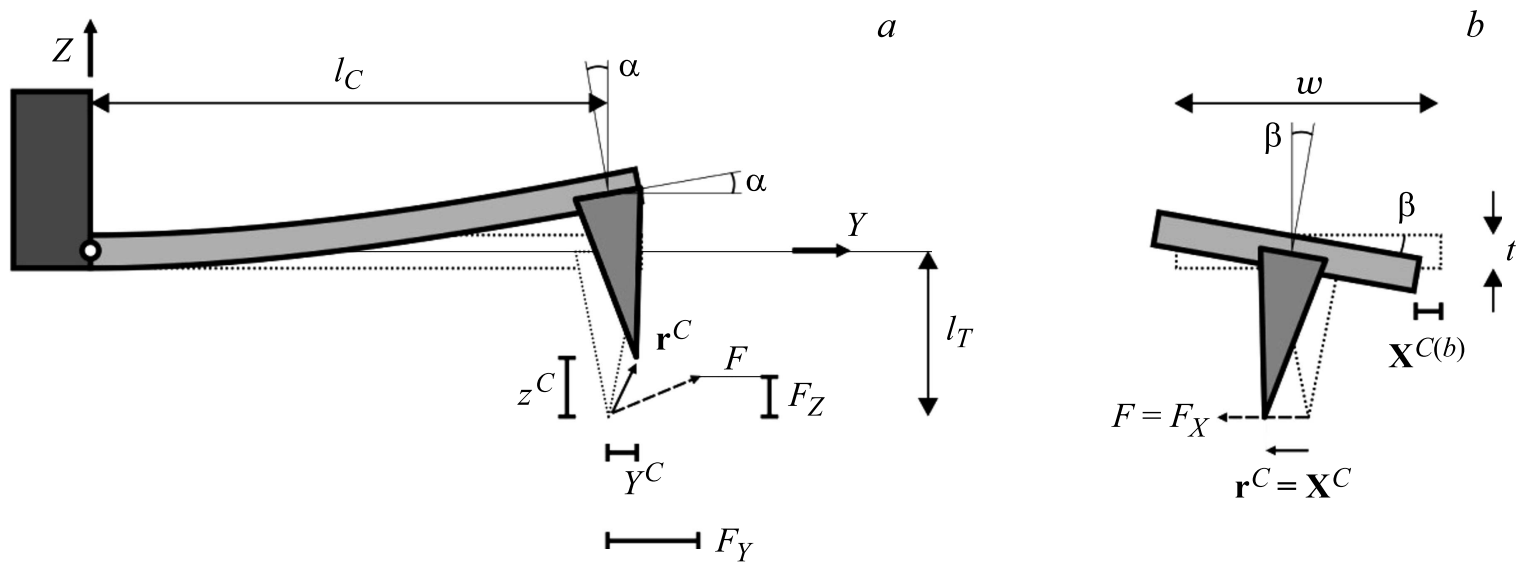

Рис. 1. „Идеальный кантилевер“: $a$ - изгиб консоли силой $F$ с компонентами $F_{Y}, F_{Z}$. И свободный конец консоли, и закрепленный на нем недеформируемый зонд отклоняются на угол $\alpha$. Кончик зонда смещается не параллельно $F$, вдоль вектора $\mathbf{r}^{C}$ с компонентами $Y^{C}, Z^{C} . b-$ сила $F=F_{X}$ закручивает консоль на угол $\beta$ и изгибает на расстояние $X^{C(b)}$. Сумма смещений кончика, вектор $\mathbf{r}^{C}=\mathbf{X}^{C}$, параллельна $F$. Обозначены характеристики: ширина $-w$, толщина $-t$, длина $-l_{C}$ консоли; высота зонда $-l_{T}$; оси $-Y, Z$ системы координат кантилевера.

на горизонтальном образце без рельефа в (1) был введен поправочный коэффициент.

В настоящей работе модель [6] использована в предположении скользящего контакта. Разработан корректирующий фильтр, аналитическое преобразование для вычислений величины $k_{S}$, и соответствующей деформации, по измеренным значениям $\sigma$ и высоты рельефа, а также характеристикам кантилевера. Показана явная зависимость преобразования от величины полярного угла локальной нормали к поверхности, которая обосновывает способ идентификации скользящего контакта АCM-зонд-образец по росту кажущейся деформации на крутых склонах рельефа образца $[7,8]$.

\section{1. Теория}

На рис. 1 рассмотрено устройство прямоугольного кантилевера, его геометрические характеристики, реакция на силу, приложенную к кончику зонда. Деформации зонда нет, анализируется так называемый „идеальный кантилевер“ [6]. Введем параметры: $\lambda=l_{T} / l_{C}-$ отношение высоты зонда к длине консоли, $k_{C}-$ жесткость консоли, $\psi=Y / l_{C}-$ нормированная координата точки фокуса АСМ-лазера ОР. Запишем связь угла изгиба консоли $\alpha$, измеряемого схемой ОР, с компонентами $Y^{C}$ и $Z^{C}$ вектора смещения „идеального кантилевера“, а именно кончика его недеформируемого зонда, $\mathbf{r}^{C}[6]$ :

$$
\alpha\left(\psi, \mathbf{r}^{C}\right)=l_{C}^{-1}\left[\psi(3 \psi-2) Y^{C} / \lambda+6 \psi(1-\psi) Z^{C}\right] .
$$

Уравнение содержит также зависимость от $\psi$, которая отвечает за профиль угла изгиба консоли „идеального кантилевера“ при заданных фиксированных смещениях кончика зонда.

Для расчета профиля угла изгиба консоли реального кантилевера надо учесть распределение деформации в системе консоль-зонд-образец (рис. 2, a).
В каждой подсистеме: консоль, „идеальный кантилевер“, $C$ (cantilever); зонд $T$ (tip); образец $S$ (sample), действует обобщенный закон Гука. Симметричные, положительно определенные тензоры жесткости $C_{i, j}, T_{i, j}$, $S_{i, j}$ с ненулевыми детерминантами связывают компоненты вектора приложенной в точке контакта силы $F_{i}^{C, T, S}$ и компоненты вектора деформации подсистемы $r_{j}^{C, T, S}$. Тензоры податливости отвечают за обратную связь. Например, для „идеального кантилевера“ $F_{i}^{C}=C_{i, j} r_{j}^{C}$ и $r_{i}^{C}=C_{i, j}^{-1} F_{j}^{C}$. Матричные элементы, например $C_{i, j}^{-1}$ в системе координат $X Y Z$ (рис. 2), имеют вид, см. также [6]:

$$
\mathbf{C}^{-1}=k_{C}^{-1}\left(\begin{array}{ccc}
2 \lambda^{2}+\delta^{2} & 0 & 0 \\
0 & 3 \lambda^{2} & 3 \lambda / 2 \\
0 & 3 \lambda / 2 & 1
\end{array}\right),
$$

где $\delta=t / w$ - отношение толщины к ширине консоли (рис. 1,b).

Если аппроксимировать зонд усеченным конусом с осью, перпендикулярной консоли, то матрица $T_{i, j}^{-1}$ будет диагональной в системе координат $X Y Z$ :

$$
\mathbf{T}^{-1}=k_{C}^{-1}\left(\begin{array}{ccc}
\kappa_{T l}^{-1} & 0 & 0 \\
0 & \kappa_{T l}^{-1} & 0 \\
0 & 0 & \kappa_{T n}^{-1}
\end{array}\right),
$$

где $\kappa_{T l}=k_{T l} / k_{C}$ и $\kappa_{T n}=k_{T n} / k_{C}, k_{T l}$ - это жесткость зонда на изгиб, $k_{T n}-$ его нормальная жесткость, вдоль высоты конуса, см. также [6].

Когда с кончиком зонда контактирует сплошной механически анизотропный образец, тензор податливости имеет диагональный вид в системе $x y z$ (рис. $2, b$ ).

$$
\mathbf{S}^{-1}=k_{C}^{-1}\left(\begin{array}{ccc}
\kappa_{x}^{-1} & 0 & 0 \\
0 & \kappa_{y}^{-1} & 0 \\
0 & 0 & \kappa_{z}^{-1}
\end{array}\right),
$$



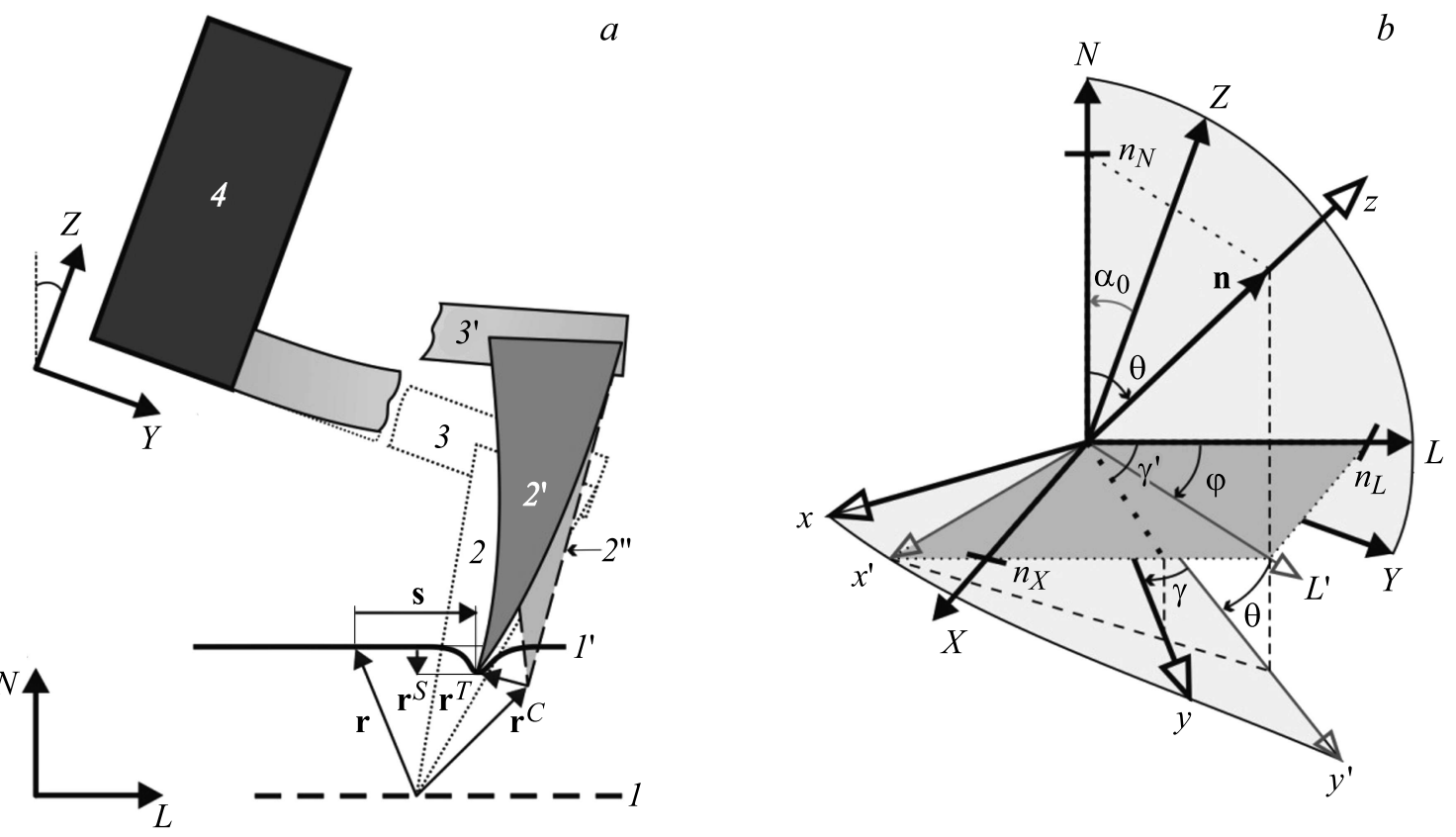

Рис. 2. $a$ - деформации АСМ-кантилевера и контактирующего с ним образца. Начальное состояние: образец (1) касается зонда (2), сила взаимодействия - нуль, консоль (3) не изогнута. Конечное состояние: образец $\left(1^{\prime}\right)$ перемещен вдоль вектора $\mathbf{r}$, деформировался сам, $\mathbf{r}^{S}$ деформировал зонд $\left(2^{\prime}\right), \mathbf{r}^{T}$, и консоль $\left(3^{\prime}\right), \mathbf{r}^{C}$; недеформируемый держатель кантилевера $(4)$ неподвижен; условно недеформируемый зонд оказывается в положении $\left(2^{\prime \prime}\right)$. Обозначены: вектор проскальзывания контакта, $\mathbf{s}$, угол установки держателя кантилевера, $\alpha_{0} . b-$ системы координат: кантилевера, $X Y Z$; сканера, $X L N$; образца, в локальной области, $x y z$, нормаль $\mathbf{n}$ к области. $X Y Z$ переводится в $X L N$ поворотом вокруг оси $X$ на угол $\alpha_{0}$. Три поворота переводят $X L N$ в $\left.x y z: 1\right)$ на угол $\varphi$ $\left.\left(X L N \rightarrow x^{\prime} L^{\prime} N\right), 2\right)$ на угол $\left.\theta\left(x^{\prime} L^{\prime} N \rightarrow x^{\prime} y^{\prime} z\right), 3\right)$ на угол $\gamma\left(x^{\prime} y^{\prime} z \rightarrow x y z\right)$. Проекция оси $y$ на плоскость сканирования $X L$ составляет с осью $L$ угол $\gamma^{\prime}, \tan \gamma=\tan \left(\gamma^{\prime}-\varphi\right) / \cos \theta$. Проекции $n_{X}, n_{L}$ и $n_{N}$, углы $\theta, \varphi$ и $\gamma^{\prime}$ определяются по данным топографии.

где $\kappa_{x, y, z}=k_{x, y, z} / k_{C}, k_{z}=k_{S}$ - локальная нормальная жесткость контакта зонд-образец, $k_{x}$ и $k_{y}$ - локальные жесткости сдвига в соответствующих направлениях.

Энергия всей системы выражается через квадратичные формы тензоров жесткости $\mathbf{C}, \mathbf{T}$ и $\mathbf{S}$ :

$$
W=1 / 2 \sum_{i, j}\left(C_{i, j} r_{i}^{C} r_{j}^{C}+T_{i, j} r_{i}^{T} r_{j}^{T}+S_{i, j} r_{i}^{S} r_{j}^{S}\right)
$$

В начальном состоянии 3/2/1 (рис. 2,a) деформаций нет; энергия, квадратичная форма (6) в минимуме. Если с помощью АСМ-сканера сместить держатель образца на фиксированный вектор $\mathbf{r}=(X, L, N)$ относительно держателя кантилевера, то в каждой подсистеме возникнут деформации: $\mathbf{r}^{C}, \mathbf{r}^{T}$ и $\mathbf{r}^{S}$. В конечном состоянии равновесия $3^{\prime} / 2^{\prime} / 1^{\prime}$ (рис. 2,a) девять компонент этих векторов должны быть координатами условного минимума квадратичной формы (6).

Пусть контакт скользит в плоскости $x y$ системы координат образца рис. $2, b$ (на рис. $2, a$ показано скольжение в плоскости $X L)$, см. также [6]. Тогда условный минимум определяется голономной связью для $z$-компонент деформаций консоли, образца и зонда:

$$
z^{C}+z^{T}-z^{S}=z=(\mathbf{r}, \mathbf{n})
$$

Например, при измерении силовых кривых сканер двигается вертикально, $\mathbf{r}=(0,0, N)$, следовательно,
$z=N \cos \theta$. С учетом (7) минимум энергии будет решением системы из восьми уравнений для восьми неизвестных, [6]. Запишем без вывода вектор решения $\mathbf{r}^{C}$ :

$$
\begin{aligned}
\mathbf{r}^{C}= & \left(x^{C}, y^{C}, z^{C}\right)=N \cos \theta\left(C_{z x}^{-1}, C_{z y}^{-1}, C_{z z}^{-1}\right) \\
& /\left(C_{z z}^{-1}+T_{z z}^{-1}+S_{z z}^{-1}\right) .
\end{aligned}
$$

Согласно (5) $S_{z z}^{-1}=\kappa_{z}^{-1}=k_{S}^{-1}$, а остальные элементы тензоров в (8) выражаются в системе $x y z$ через компоненты (3) и (4), записанные в системе $X Y Z$. Нужное для этого преобразование $X Y Z \rightarrow x y z$ дает комбинация поворотов (рис. 2,b). Обратное преобразование $x y z \rightarrow X Y Z$ позволяет через компоненты $\mathbf{r}^{C}$ выразить деформации $Y^{C}$ и $Z^{C}$, определяющие угол изгиба консоли в выражении (2).

Комбинация (8) и (2) дает отношение угла изгиба консоли $\alpha\left(\psi, \mathbf{r}^{C}\right)$ к вертикальному перемещению сканера $N$. Получается модельный наклон силовой кривой $S$. Если взять $k_{S}^{-1}=0$ и направить ось $\mathbf{z}$ вертикально, $\mathbf{n} \| \mathbf{N}$, (рис. $2, b)$, то моделируется $S_{0}$. Поэтому можно записать

$$
\alpha\left(\psi, \mathbf{r}^{C}\right) / \alpha\left(\psi,\left.\mathbf{r}^{C}\right|_{k_{S}^{-1}=0, \mathbf{n} \| \mathbf{N}}\right)=\sigma
$$

С помощью (9) $k_{S}$ выражается через относительный наклон силовых кривых $\sigma$, углы $\theta$ и $\varphi$, характеризующие локальный рельеф образца, угол установки держателя 
Коэффициенты $A, B, C$ в уравнениях (11a)-(12)

\begin{tabular}{|c|c|c|}
\hline \multicolumn{3}{|c|}{$A=\left[\left(1+\kappa_{T n}^{-1}\right) a_{0}^{2}+\left(3 \lambda^{2}+\kappa_{T l}^{-1}\right) b_{0}^{2}-3 \lambda a_{0} b_{0}\right][(2-\psi) a-2 \lambda b] /\left[(2-\psi) a_{0}-2 \lambda b_{0}\right]$} \\
\hline \multicolumn{3}{|c|}{$B=\left(1+\kappa_{T n}^{-1}\right) a^{2}+\left(3 \lambda^{2}+\kappa_{T l}^{-1}\right) b^{2}-3 \lambda a b+\left(2 \lambda^{2}+\delta^{2}+\kappa_{T l}^{-1}\right) N_{X}^{2}$} \\
\hline \multicolumn{3}{|c|}{$C=1 /\left(1+N_{X}^{2}+N_{L}^{2}\right)=\cos ^{2} \theta$} \\
\hline$a=\cos \alpha_{0}-N_{L} \sin \alpha_{0}$ & $b=\sin \alpha_{0}+N_{L} \cos \alpha_{0}$ & $b_{0}=\sin \alpha_{0}$ \\
\hline
\end{tabular}

кантилевера $\alpha_{0}$, параметры кантилевера $\lambda, \delta, k_{C}$ и зонда $k_{T n}, k_{T l}$. Определение параметра жесткости зонда на сдвиг $k_{T l}$ по петлям трения было рассмотрено в [6]. Если линейно связать $k_{T n}$ с $k_{T l}$, то все три переменных и семь параметров в $k_{S}\left(\sigma, \theta, \varphi, \psi, \alpha_{0}, \lambda, \delta, k_{C}, k_{T n}, k_{T l}\right)$ оказываются заданными.

Например, переменные углы $\theta$ и $\varphi$ (рис. 2, $b$ ), характеризующие нормаль в точке интереса на образце, вычисляются по данным АСМ-топографии. Высота рельефа $N=N(X, L)$ зависит от координат $X$ и $L$ в плоскости сканирования. Производные $N(X, L) / \partial X=N_{X}$ и $N(X, L) / \partial L=N_{L}$ в точке $(X, L, N)$ определяют компоненты $\mathbf{n}$ и искомые $\varphi$ и $\theta$ :

$$
\begin{gathered}
\mathbf{n}=\left(-N_{X},-N_{L}, 1\right) / \sqrt{1+N_{X}^{2}+N_{L}^{2}} \\
\cos \varphi=-N_{L} / \sqrt{N_{X}^{2}+N_{L}^{2}}, \cos \theta=1 / \sqrt{1+N_{X}^{2}+N_{L}^{2}}
\end{gathered}
$$

В зависимость для $k_{S}$ не входит четвертая переменная, угол $\gamma$. Согласно рис. $2, b$, переход $x^{\prime} y^{\prime} z \rightarrow x y z$ осуществляется поворотом вокруг оси $z$ на угол $\gamma$. Так как матричный элемент $S_{z z}^{-1}$ в (5) инвариантен к таким вращениям, в качестве системы координат образца выбрана $x^{\prime} y^{\prime} z$, что сократило число переменных.

C помощью системы компьютерной алгебры Mathcad был определен аналитический вид зависимости $\kappa_{S}^{-1}$ $\left(\kappa_{A}^{-1}, N_{X}, N_{L}, \psi, \alpha_{0}, \lambda, \delta, \kappa_{T n}^{-1}, \kappa_{T l}^{-1}\right):$

$$
\kappa_{S}^{-1}=C A \sigma^{-1}-C B=C\left(A \kappa_{A}^{-1}+A-B\right),
$$

см. также выражения для коэффициентов в таблице. Для горизонтального образца без рельефа $N_{X}=N_{L}=0$. С помощью таблицы получаем $A=B=A_{0}, \quad C=1$ и (11a) в виде $\kappa_{S}=A_{0}^{-1} \kappa_{A}$. Предложенный в [6] поправочный коэффициент совпадает с $A_{0}^{-1}$.

Уравнение (11a) можно рассматривать как корректирующее преобразование, фильтр для вычислений нормальной относительной податливости образца $\kappa_{S}^{-1}$ по измеренным значениям $\kappa_{A}^{-1}$ и данным топографии рельефа, а также характеристикам кантилевера. При АСМсканировании с постоянной силой взаимодействия произведение силы на $k_{C}^{-1} \kappa_{S}^{-1}$ дает корректные значения локальной деформации образца. Учитывая ее в высоте рельефа, можно уточнить значения $N_{X}$ и $N_{L}$, а потом применить (11a) для следующего приближения $\kappa_{S}^{-1}$. Эта процедура уместна на мягком образце, таком как живая клетка, когда измеренные при АСМ-сканировании деформация и высота рельефа сопоставимы.

С другой стороны,

$$
\kappa_{A}^{-1}=\kappa_{S}^{-1} / C A-1+B / A .
$$

Это уравнение удобно для моделирования АСМ-сигнала. Возьмем, к примеру, мягкий модельный образец постоянной податливости, $\kappa_{S}^{-1} \gg 1$. Поскольку $B / A \sim 1$, (11б) переходит в приблизительное равенство

$$
\kappa_{A}^{-1} \approx \kappa_{S}^{-1} / C A \sim \cos ^{-2} \theta .
$$

Такая зависимость $\kappa_{A}^{-1}$ от $\theta$, полярного угла локальной нормали к поверхности, означает, что измеряемый в АСМ сигнал деформации (он пропорционален $\kappa_{A}^{-1}$ ) должен возрастать на крутых склонах мягкого однородного образца. Это следствие обосновывает способ идентификации скольжения АСМ-зонда по образцу, предложенный ранее $[7,8]$.

\section{2. Эксперимент}

C помощью атомно-силового микроскопа BioScope Catalyst (Bruker, США) исследовались два тестовых образца. Измерения велись в режиме АСМ PeakForce QNM, позволяющем одновременно визуализировать сигналы высоты рельефа образца, деформации и рассогласования (ошибки регулирования пиковой силы). Сигналы определялись автоматически алгоритмом анализа силовых кривых, встроенным в программу сканирования. Обработка результатов измерений осуществлялась с помощью программ для визуализации и анализа данных сканирующей зондовой микроскопии NanoScope Analysis v1.80, Gwyddion 2.55 и системы компьютерной алгебры Mathcad15.

Для вычисления значений $N_{X}$ и $N_{L}$ в программе Gwyddion к данным изображений рельефа образцов применялся горизонтальный или вертикальный фильтр Собеля. Кроме того, нормировался автоматически детектируемый, пропорциональный пиковой силе сигнал деформации $D_{E}$, используя следующее выражение:

$$
D=D_{E} F_{\text {SetPoint }} /\left(F_{\text {SetPoint }}+F_{\text {Error }}\right),
$$

где $D-$ нормированное значение деформации, $F_{\text {SetPoint }}$ - заданное значение пиковой силы, а $F_{\text {Error }}-$ локальное отклонение от $F_{\text {SetPoint }}$. Нормировка нужна 

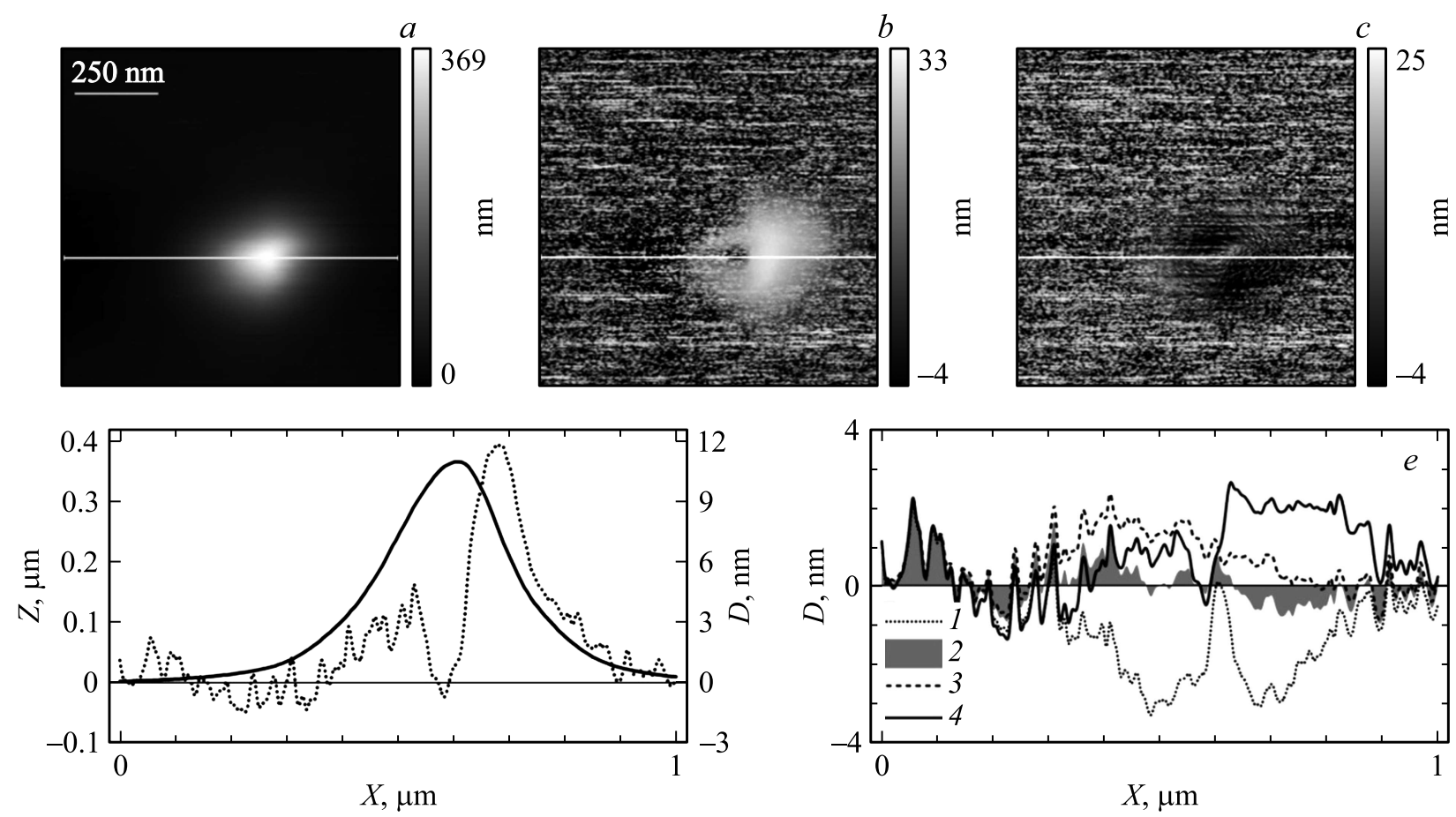

Рис. 3. $a-$ тоновая топография острия решетки TGT1. Карты сигнала деформации: $b-$ нормированного исходного; $c-$ после оптимальной коррекции предложенным в работе фильтром; $d$ - профили высоты (сплошная кривая) и деформации (точечная) вдоль сечений сигналов на $a$ и $b ; e$ - профили скорректированной деформации: $1, k_{T l}=10 \mathrm{~N} / \mathrm{m} ; 2, k_{T l}=32 \mathrm{~N} / \mathrm{m}$, сечение на $c ; 3, k_{T l} \rightarrow \infty ; 4$, исходной, умноженной на $(\cos \theta)^{2}$. Параметры: РеakForce QNM режим; пиковая сила - $60 \mathrm{nN}$, частота вертикального зондирования $-1 \mathrm{kHz}$, амплитуда $-50 \mathrm{~nm}$; сканирование - справа налево с частотой $-0.4 \mathrm{~Hz}$; кантилевер FMG01, угол установки держателя кантилевера $\alpha_{0}=10^{\circ}, k_{C}=2.76 \mathrm{~N} / \mathrm{m}$ (метод тепловых шумов), $\lambda=12.5 / 225, \delta=2.5 / 32$.

для корректного вычисления кажущейся относительной податливости $\kappa_{A}^{-1}=k_{C} D / F_{\text {SetPoint }}$. Полученные массивы значений $N_{X}, N_{L}$ и $D$ загружались в программу Mathcad для обработки по формуле (11a).

Результаты АСМ-исследования тестового образца TGT1 (НТ-МДТ СИ, Россия) приведены на рис. 3. TGT1 - это периодический массив острых кремниевых иголок с углом при вершине $50 \pm 10^{\circ}$ (полярный угол $-\theta \approx 65^{\circ}, \cos ^{-2} \theta \approx 6$ ). Если предположить, что контактная жесткость зонд-острие существенно меньше жесткости консоли, то вокруг острия на АCMизображении сигнала кажущейся деформации должно быть симметричное гало. На рис. 3, $b$ гало наблюдается, но асимметричное. Сопоставление профилей высоты и кажущейся деформации на рис. $3, d$ показывает, что точно на вершине острия деформация не детектируется.

В этих измерениях вдоль горизонтальной стороны кадра была ось $Y$ кантилевера FMG01, его держатель располагался слева. С учетом наклона держателя консоль составляла с левой и правой сторонами острия не равные углы - примерно 75 и $55^{\circ}$ соответственно, что качественно объясняет асимметрию гало в сигнале кажущейся деформации.

На рис. 3, с показаны результаты оптимальной фильтрации сигнала деформации, $k_{T l}^{\mathrm{opt}}=32 \mathrm{~N} / \mathrm{m}$. Варьировался один параметр зонда, $k_{T l}$, второй был связанным:
$k_{T n}=40 k_{T l}$. Такой коэффициент пропорциональности выбран по оценкам нормальной и сдвиговой жесткости АСМ-зонда, [6]. Оптимальная фильтрация обеспечивала на всем изображении минимум среднеквадратичного отклонения скорректированной деформации. Изображение на рис. 3, $с$ и профиль 2 на рис. 3, $е$ показывают достаточно симметричный сигнал вокруг острия со средним нулевым значением деформации. Для $k_{T l}<k_{T l}^{\text {opt }}$ (профиль 1 рис. $3, e)$ вокруг острия наблюдается отрицательная деформация, лишенная, в рамках модели для фильтра, физического смысла. Когда $k_{T l} \rightarrow \infty$ (профиль 3 рис. 3,e) область вокруг и само острие показывают положительную деформацию, $1-2 \mathrm{~nm}$, сопоставимую с шумом. Если коррекция учитывает только вклад полярного угла (профиль 4 рис. $3, e$ ), то сигнал падает почти на порядок, но гало остается асимметричным. В дальнейшем, опираясь на эти результаты, оптимальной считалась фильтрация с минимальным среднеквадратичным отклонением скорректированной деформации.

На рис. 4 показаны результаты АСМ-исследования наномостика, образованного нанотубулярным гидросиликатом [9] над углублением решетки TGZ03 (НТ-МДТ СИ, Россия). По величине деформации мостика от известной нагрузки можно рассчитать модуль Юнга материала $[10,11]$. Как отмечалось в [11], на результат расчета критически влияет не только 

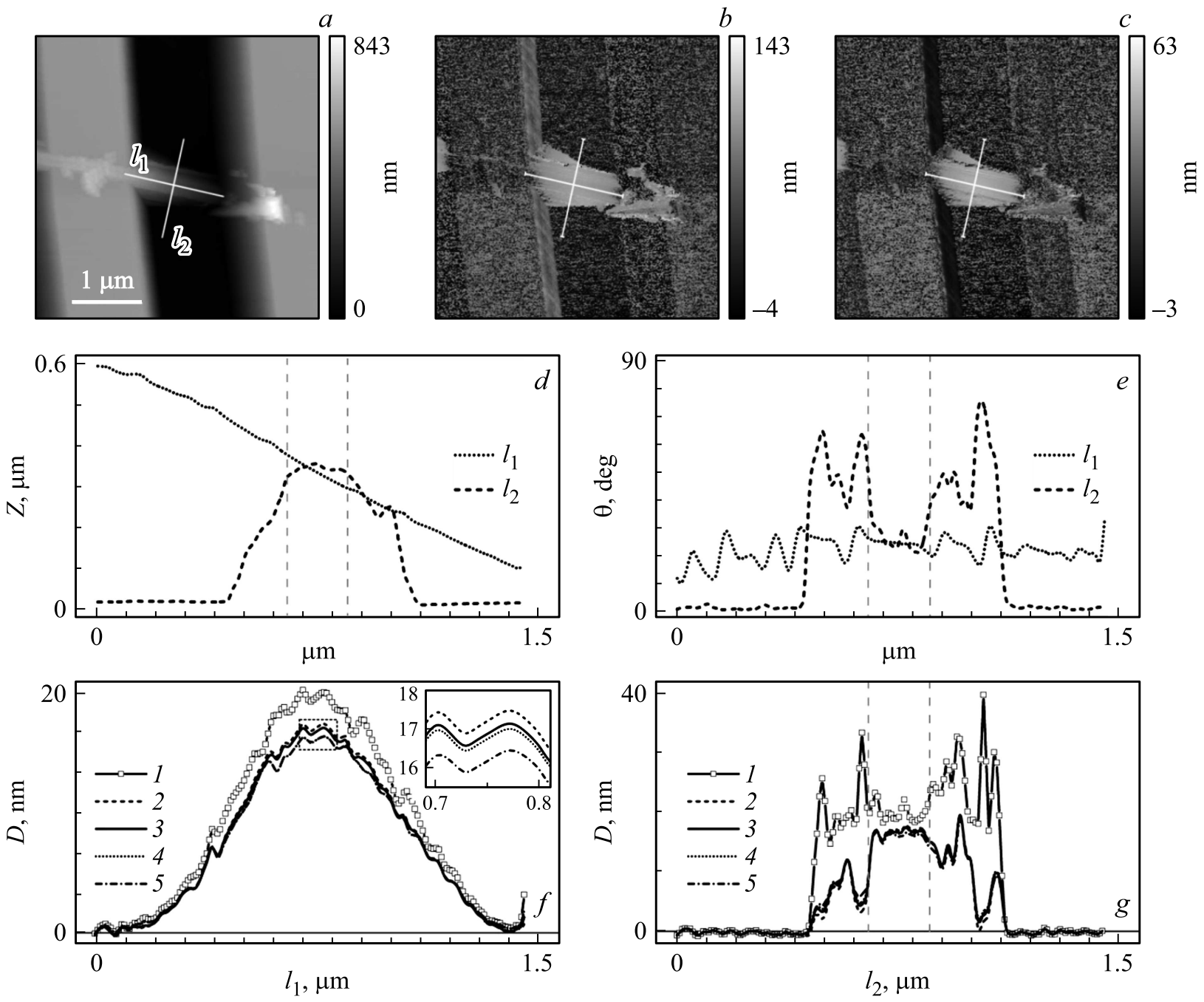

Рис. 4. $a-$ тоновая топография наномостика. Карты сигнала деформации: $b-$ нормированного исходного, $c-$ после оптимальной коррекции. $d-$ профили высоты вдоль и поперек наномостика, см. линии сечений на $a$; $e-$ профили полярного угла, соответствующие сечениям на $a . f$ и $g$ - профили деформации вдоль и поперек наномостика. Исходный сигнал - 1 . После коррекции: $2, k_{T l}=4 N / m ; 3, k_{T l}=12 N / m$, сечение на $c ; 4, k_{T l} \rightarrow \infty ; 5$, после умножения на $(\cos \theta)^{2}$. В правом верхнем углу $f$ вставка с увеличенным изображением хода скорректированных сигналов в максимуме. Вертикальные пунктирные линии на $d$, $e$ и $g$ отмечают края наномостика на поперечных сечениях. Параметры: $10 \mathrm{nN}, 1 \mathrm{kHz}, 150 \mathrm{~nm}$; справа налево, $0.2 \mathrm{~Hz} ; \mathrm{FMG01,} \alpha_{0}=10^{\circ}$ $k_{C}=2.384 \mathrm{~N} / m, \lambda=12.5 / 225, \delta=2.5 / 32$.

максимальная амплитуда, но и форма профиля деформации наномостика при фиксированной нагрузке. В частности, в [11] предложен алгоритм определения условий закрепления наномостика по форме профиля деформации. Изображение с наномостиком (рис. 4, $a$ ), профили высоты и угла $\theta$ вдоль наномостика, сечения $l_{1}$ (рис. $4, d, e$ ) показывают, что последний наклонен примерно на $25^{\circ}$. Рис. $4, f$ с поведением деформации вдоль наномостика показывает, что сигнал после коррекции уменьшается примерно на 15\%. Как следствие, на $15 \%$ увеличивается определяемый модуль Юнга, E. Минимальная жесткость наномостика $k_{\min }=(10 / 17) N / m$, диаметр $d=35 \mathrm{~nm}$ (слабо заметное двоение изображения на рис. $4, a$ вызвано несовершенством формы АСМ-зонда, электронная микроско- пия показала, что мостик одинарный), длина пролета $l=1300 \mathrm{~nm}$. Если он защемлен на решетке, расчет дает $E=k_{\min } l^{3} /\left(3 \pi d^{4}\right) \cong 90 \mathrm{GPa}$, величину, характерную для материала наномостика [11].

Интересно выяснить условия закрепления, изучая профиль сигнала деформации, взятый поперек наномостика. Если наномостик опирается на решетку, то при соскакивании зонда с наномостика сигнал деформации должен возрастать. На рис. 4, g края наномостика отмечены вертикальными пунктирными линиями. Вне линий сигнал исходной деформации (профиль 1 ) заметно больше, чем между ними. Однако скорректированные профили дают обратную картину. Поэтому сделанное предположение, скорее, не верно. Для более точного вывода требуется применить алгоритм, предложенный в [11]. 
Стоит добавить, что скорректированные профили 2-4 на рис. $4, f$ практически совпадают и лишь незначительно отличаются от профиля 5. Это ожидаемый результат, так как минимальная жесткость наномостика почти на порядок меньше жесткости примененного кантилевера, а в профиле 5 учтен вклад $(\cos \theta)^{-2}$, и остался лишь незначительный вклад от $\alpha_{0}$.

\section{Заключение}

Для скользящего контакта АСМ-зонда по поверхности с произвольным рельефом разработан фильтр корректировки сигналов контактной жесткости зонд-образец, деформации, а также высоты рельефа.

Фильтр использован для обработки сигнала деформации в двух тестовых АСМ-экспериментах. Для сохранения простоты величина деформации образца считалась пропорциональной силе нагрузки, уравнение (13). При необходимости учесть нелинейности в зависимости силы от деформации следует фильтровать сигналы дифференциальной жесткости или дифференциальной податливости.

Показана эффективность применения фильтра для совершенствования АСМ-измерений методом испытаний на изгиб модуля Юнга наномостиков, не лежащих в плоскости сканирования.

Результаты работы важны для повышения точности наномеханических АСМ-измерений, в частности, в исследованиях мягких объектов, таких как живые клетки.

\section{Финансирование работы}

Исследование выполнено при поддержке Российского научного фонда, грант № 19-13-00151.

\section{Конфликт интересов}

Авторы заявляют, что у них нет конфликта интересов.

\section{Список литературы}

[1] Sarid D. Exploring Scanning Probe Microscopy with MATHEMATICA. Second edition. Weinheim: Wiley-VCH Verlag $\mathrm{GmbH} \&$ Co. KGaA, 2007. 310 p.

[2] Анкудинов А.В., Халисов М.М., Пеннияйнен В.А., Подзорова С.А., Тимошук К.И., Крылов Б.В. // Письма в ЖТФ. 2018. Т. 44. Вып. 15. С. 38-45.

DOI: 10.21883/PJTF.2018.15.46438.17351

[Ankudinov A.V., Khalisov M.M., Penniyaynen V.A., Podzorova S.A., Timoshchuk K.I., Krylov B.V. // Tech. Phys. Lett. 2018. Vol. 44. P. 671-674.

DOI: $10.1134 / \mathrm{S} 1063785018080035]$

[3] Fujisawa S., Ohta M., Konishi T., Sugawara Ya., Morita S. // Rev. Sci. Instrum. 1994. Vol. 65. N 3. P. 644-647.

DOI: $10.1063 / 1.1145131$

[4] Kawakatsu H., Bleuler H., Saito T., Hiroshi K. // Jpn. J. Appl. Phys. 1995. Vol. 34. Pt. 1. N 6B. P. 3400-3402.

DOI: 10.1143/JJAP.34.3400
[5] Asylum Research Quantifies the „Last Axis“ in Atomic Force Microscopy. [Электронный pecypc] 09.02.2018. URL: https://www.oxford-instruments.com

[6] Ankudinov A.V. // Nanosystems: Physics, Chemistry, Mathematics. 2019. Vol. 10. N 6. P. 642-653. DOI: $10.17586 / 2220-8054-2019-10-6-642-653$

[7] Тимощук К.И., Халисов М.М., Пеннияйнен В.А., Крылов Б.В., Анкудинов А.В. // Письма в ЖТФ. 2019. Т. 45. Вып. 18. C. 44-47. DOI: 10.21883/PJTF.2019.18.48238.17878 [Timoshchuk K.I., Khalisov M.M., Penniyaynen V.A. Krylov B.V., Ankudinov A.V. // Tech. Phys. Lett. 2019. Vol. 45. N 9. P. 947-950. DOI: 10.1134/S1063785019090293]

[8] Автореф. канд. дис. Тимощук К.И. Методики исследования мягких объектов в атомно-силовой микроскопии. Санкт-Петербургский национальный исследовательский университет информационных технологий, механики и оптики. СПб., 2019. 18 с.

[9] Krasilin A.A., Nevedomsky V.N., Gusarov V.V. // J. Phys. Chem. 2017. Vol. 121. N 22. P. 12495-12502. DOI: 10.1021/acs.jpcc.7b03785

[10] Salvetat J.-P., Kulik A.J., Bonard J.-M., Briggs G.A.D., Stockli T., Metenier K., Bonnamy S., Beguin F., Burnham N.A., Forro L. // Adv. Mater. 1999. Vol. 11. P. 161165. DOI: 10.1002/(SICI)1521-4095(199902)11:2<161::AIDADMA161>3.0.CO;2-J

[11] Ankudinov A.V. // Semiconductors. 2019. Vol. 53. N 14. P. $1891-1899$. DOI: $10.1134 / \mathrm{s} 1063782619140021$ 